$\mathrm{PM} / 01-14$

hep-ph/0103270

\title{
Comment on "Infrared Fixed Point Structure in Minimal Supersymmetric Standard Model with Baryon and Lepton Number Violation"
}

\author{
Y. MAMBrini ${ }^{a}$, G. MoultakA ${ }^{b}$ \\ a $C E A / D I F / D P T A / S P N$ \\ B.P. 12 F-91680 Bruyères-le-Châtel, France \\ ${ }^{b}$ Physique Mathématique et Théorique, UMR No 5825-CNRS, \\ Université Montpellier II, F-34095 Montpellier Cedex 5, France.
}

\begin{abstract}
We reconsider the Infrared Quasi Fixed Points which were studied recently in the literature in the context of the Baryon and Lepton number violating Minimal Supersymmetric Standard Model (hep-ph/0011274). The complete analysis requires further care and reveals more structure than what was previously shown. The formalism we develop here is quite general, and can be readily applied to a large class of models.
\end{abstract}

Submitted to Phys.Rev. D 
Scenarios with R-parity violation, and in particular baryon or lepton number violation within the Minimal Supersymmetric Standard Model ( $\not R_{p}$-MSSM), have attracted attention in the past few years. In a recent work, Ananthanarayan and Pandita have carried out a study of the Infrared Fixed Point structure of baryon/lepton number violating couplings in such scenarios [1] . Issues related to the Infrared Fixed Points, and in particular to the Infrared Quasi Fixed Points (IRQFP) of the running Yukawa type couplings, can provide generic information about the electroweak scale physics irrespectively of any high energy physics assumptions. While IRQFPs are always present due to the Landau pole in the running of the Yukawa couplings, or equivalently due to a perturbativity bound on those couplings, exact fixed points occur only in physically simplified configurations (projecting on some subset of Yukawa couplings and neglecting some gauge couplings). Of course when exact fixed points exist it is interesting to study their relation to the IRQFP as is done in [1]. However one should keep in mind that such a relation need not exist theoretically, not to mention that one expects anyway the IRQFP regime to be physically more relevant than the one due to exact fixed points, as was initially shown in [2]. In the present comment we concentrate exclusively on the IRQFP structure. In ref. [1], the general analytical forms for the Yukawa couplings, derived in [3], were applied to the special case of the $R_{p}$-MSSM with the following (squared) running Yukawa couplings $\tilde{Y}_{t}, \tilde{Y}_{b}, \tilde{Y}_{\tau}, \tilde{Y}, \tilde{Y}^{\prime}, \tilde{Y}^{\prime \prime}$ (see [1] for notations). These analytical expressions contain denominators of the form $1+a_{j j} \tilde{Y}_{j}(0) \int_{0}^{t}(\ldots)_{j}$. The authors of [1] then claimed that "in the regime where the Yukawa couplings $\tilde{Y}_{t}(0), \tilde{Y}_{b}(0), \tilde{Y}_{\tau}(0), \tilde{Y}(0), \tilde{Y}^{\prime}(0), \tilde{Y}^{\prime \prime}(0) \rightarrow \infty$ with their ratios fixed, it is legitimate to drop 1 in the denominators..." . This is in general a wrong statement. "Neglecting 1 " can happen to be justified in special cases, but this can be seen only a posteriori and at the expense of a more careful study. For instance it was indeed proven to be correct in the R-parity conserving MSSM $\left(R_{p}\right.$-MSSM) in the $t / b / \tau$ sector [4], but to be incorrect in the next to minimal $R_{p}$-MSSM [5]. The reason is simple to understand from the analytical structure involved. The relevant Renormalization Group Equations (RGE) governing the running of the Yukawa type couplings to one-loop order are of the form

$$
\frac{d}{d t} \tilde{Y}_{k}(t)=\tilde{Y}_{k}(t)\left(\sum_{i} c_{k i} g_{i}^{2}(t)-\sum_{l} a_{k l} \tilde{Y}_{l}(t)\right)
$$

where $t$ denotes the scale evolution parameter, $\tilde{Y}_{k}$ the squared Yukawa couplings, $g_{i}^{2}$ the squared gauge couplings, and where $c_{k i}$ and $a_{k l}$ are constant coefficients depending on the model.

The general solution for such a system (valid for any number of Yukawa couplings labeled by $k$ ) reads [3]

$$
\tilde{Y}_{k}(t)=\frac{\tilde{Y}_{k}(0) u_{k}(t)}{1+a_{k k} \tilde{Y}_{k}(0) \int_{0}^{t} u_{k}\left(t^{\prime}\right) d t^{\prime}}
$$

where the auxiliary functions $u_{k}$ are given by

$$
u_{k}(t)=\frac{E_{k}(t)}{\prod_{j \neq k}\left(1+a_{j j} \tilde{Y}_{j}(0) \int_{0}^{t} u_{j}\left(t^{\prime}\right) d t^{\prime}\right)^{a_{k j} / a_{j j}}}
$$

and the functions $E_{k}(t) \equiv \exp \left[\int_{0}^{t} \sum_{i} c_{k i} g_{i}^{2}\left(t^{\prime}\right) d t^{\prime}\right]$ are fully determined by the well-known running gauge couplings which need not be written more explicitly here. In the case of [四], $k=1, \ldots, 6$ 
and the $u_{k}$ 's correspond to $F_{t}, F_{b}, F_{\tau}, F, F^{\prime}, F^{\prime \prime}$ studied therein. It is easy to see from the structure of Eq.(3) that when some of (or all) the initial values $\tilde{Y}_{k}(0) \equiv Y^{0} \rightarrow \infty$, the $u_{k}$ 's should have the form

$$
u_{k} \equiv \frac{u_{k}^{\mathrm{QFP}}}{\left(Y^{0}\right)^{p_{k}}}
$$

where the $u_{k}^{\mathrm{QFP}}$ 's are initial condition independent functions, $p_{k} \geq 0$ a set of values which depend on the model under consideration and on the choice of the subset of Yukawas having large initial conditions. When for a given $j, \tilde{Y}_{j}(0) \rightarrow \infty$, it is obvious that " 1 " can be safely dropped in $1+a_{j j} Y_{j}^{0} \int_{0}^{t} u_{j}\left(t^{\prime}\right) d t^{\prime}$ only if $p_{j}<1$, while if $p_{j}>1$, "1" becomes the leading contribution, the $j^{t h}$ sector then drops out completely from the expression of $u_{k}$ in (3) and the IRQFP value of $\tilde{Y}_{j}(t) \rightarrow 0$. It can also happen that $p_{j}=1$, in which case discarding "1" requires the knowledge of the actual numerical contribution of the $Y^{0}$ independent term $a_{j j} \int_{0}^{t} u_{j}^{\mathrm{QFP}}$.

We turn now to the determination of the powers $p_{k}$ in the case of $\not R_{p}$-MSSM. This is necessary for a correct study of the IRQFP regimes and has been overlooked in ref. [1]. We start off from Eqs.(18-23) of [四].

Denoting by $p_{t}, p_{b}, p_{\tau}, p_{0}, p_{1}, p_{2}$ the powers corresponding respectively to $F_{t}, F_{b}, F_{\tau}, F, F^{\prime}, F^{\prime \prime}$, one obtains the following system,

$$
\left(\begin{array}{l}
p_{t} \\
p_{b} \\
p_{\tau} \\
p_{0} \\
p_{1} \\
p_{2}
\end{array}\right)=\left(\begin{array}{cccccc}
0 & \frac{1}{6} & 0 & 0 & \frac{1}{6} & \frac{1}{3} \\
\frac{1}{6} & 0 & \frac{1}{4} & 0 & 1 & \frac{1}{3} \\
0 & \frac{1}{2} & 0 & 1 & \frac{1}{2} & 0 \\
0 & 0 & 1 & 0 & \frac{1}{2} & 0 \\
\frac{1}{6} & 1 & \frac{1}{4} & \frac{1}{4} & 0 & \frac{1}{3} \\
\frac{1}{3} & \frac{1}{3} & 0 & 0 & \frac{1}{3} & 0
\end{array}\right)\left(\begin{array}{c}
\left(1-p_{t}\right) \theta\left[1-p_{t}\right] \delta_{t} \\
\left(1-p_{b}\right) \theta\left[1-p_{b}\right] \delta_{b} \\
\left(1-p_{\tau}\right) \theta\left[1-p_{\tau}\right] \delta_{\tau} \\
\left(1-p_{0}\right) \theta\left[1-p_{0}\right] \delta_{0} \\
\left(1-p_{1}\right) \theta\left[1-p_{1}\right] \delta_{1} \\
\left(1-p_{2}\right) \theta\left[1-p_{2}\right] \delta_{2}
\end{array}\right)
$$

where the Heaviside $\theta$-function $\left(\theta\left(x_{>0}, x_{<0}\right)=(1,0)\right)$ accounts for the possibility of the $p_{i}$ 's being larger or smaller than one, and $\delta_{n}(n=t, b, \tau, 0,1,2)$ takes the value $0(1)$ when the corresponding initial $\tilde{Y}_{n}(0)$ is finite (infinite). We illustrate in table 1 the solutions of Eq.(5) for three different sets of values of the $\delta_{n}$ 's. In the first case $\delta_{t, b, 2}=1$, that is the initial conditions for only $\tilde{Y}_{t}, \tilde{Y}_{b}, \tilde{Y}^{\prime \prime}$ are taken infinitely large. This corresponds to the case studied in 11 where $\tilde{Y}_{\tau}, \tilde{Y}, \tilde{Y}^{\prime}$ were put to zero in order to study the exact (attractive) IR fixed point. As one can see from the table, all the powers but $p_{1}(=1)$ turn out to be strictly smaller than one, so that "1" can indeed be safely neglected in the corresponding contributions. Moreover, since in the case we consider $\tilde{Y}^{\prime}(0)$ is not taken infinitely large, the contribution of $F^{\prime}$ will drop out anyway, being suppressed by a factor $1 /\left(Y^{0}\right)^{p_{1}}$ where $Y^{0}$ is the large initial condition. So in retrospect, the legitimacy of dropping "1" in this case was a lucky situation. [The reason is that the reduced matrix of the system $p_{t}, p_{b}, p_{\tau}$ satisfies a sufficient condition to forbid $p_{i}>1$, namely that the sum of the matrix elements in each row is less than 1.] We thus agree with the authors of ref. [1] on this part of their study, including the numerical estimate of the IRQFP values. The point is that such configurations do not prevail for more general initial conditions. In the second case of table 1, where we keep finite either $\tilde{Y}_{\tau}(0)$ or $\tilde{Y}^{\prime}(0)$ (but not necessarily both), $p_{\tau}, p_{1}$ are found to be both greater than one. Clearly here, neglecting one in

1 There are unfortunately some misprints in these equations, the relevant ones for us are: (1) In Eq.(19), the power of the third term in the denominator should read 1 instead of $1 / 6,(2)$ In Eq.(21) $\tilde{Y}^{\prime \prime}, F^{\prime \prime}$ should read $\tilde{Y}^{\prime}, F^{\prime}$ (in their Eq.(8) $\lambda_{233}^{\prime \prime}$ should read $\lambda_{333}^{\prime}$ ). 


\begin{tabular}{|c|c|c|c|c|c|c|}
\hline & $p_{t}$ & $p_{b}$ & $p_{\tau}$ & $p_{0}$ & $p_{1}$ & $p_{2}$ \\
\hline$\delta_{t, b, 2}=1$ & $5 / 17$ & $5 / 17$ & $6 / 17$ & 0 & 1 & $8 / 17$ \\
\hline$\delta_{\tau \text { or } 1}=0$ & $5 / 17$ & $5 / 17$ & $23 / 17$ & 0 & $5 / 4$ & $8 / 17$ \\
\hline $\mathbf{( 1 )} \delta_{\text {all }}=1$ & $5 / 17$ & $5 / 17$ & $23 / 17$ & 0 & $5 / 4$ & $8 / 17$ \\
\hline $\mathbf{( 2 )} \delta_{\text {all }}=1$ & $41 / 146$ & 1 & $18 / 73$ & 1 & $37 / 173$ & $59 / 146$ \\
\hline
\end{tabular}

Table 1: solutions for the powers in three different IRQFP regimes.

Eq.(河) in the $\tau$ sector, when $\tilde{Y}_{\tau}(0) \rightarrow \infty$, (or in the "prime" sector when $\tilde{Y}^{\prime}(0) \rightarrow \infty$ ) leads to a wrong result! Moreover, $p_{\tau}, p_{1}>1$ has a physically interesting consequence: the IRQFP values $\tilde{Y}_{\tau}^{\mathrm{QFP}}(t), \tilde{Y}^{\prime \mathrm{QFP}}(t)$ are zero, as can been seen from Eqs.(目, 目). This is at variance with what Eq.(39) of ref. [1] would have implied. There is thus a much richer structure than stated in [1], in the sense that the Yukawa couplings in the IRQFP regime can be naturally driven to the hyperplane which has the exact infrared fixed points without a prior projection on this hyperplane as an initial condition. This behavior is actually more general than in the case $\delta_{t, b, 2}=1$. It holds even when all initial conditions are taken infinite $\left(\delta_{\text {all }}=1\right)$. The latter case, considered in [1], is even more tricky to handle, since it leads to two different sets of solutions for Eq.(5) as can be seen from table 1. These solutions correspond to two different behaviors in the IRQFP limit. However, due to the uniqueness of the running Yukawa's for given initial conditions (no singularities are encountered when running from the GUT scale down to the electroweak scales), one of these solutions should be spurious. The correct solution turns out to be (1). This is found consistently, both from analytical considerations which we do not describe here, and from a numerical study illustrated in figure 1. Solution (2) would have required all the Yukawa couplings to become $\tilde{Y}^{0}$ independent when the latter goes increasingly large, while in solution (1) the couplings $\tilde{Y}^{\prime}$ and $\tilde{Y}_{\tau}$ are expected to decrease to zero. Figure 1 exhibits clearly the features of solution (1), where the $\operatorname{logs}$ of $\tilde{Y}^{\prime}$ and $\tilde{Y}_{\tau}$ decrease linearly as expected and with rates close to the expected ones (respectively .25 and .35 ). The behavior of $\tilde{Y}^{\prime \mathrm{QFP}}$ and $\tilde{Y}_{\tau}^{\mathrm{QFP}}$ contradicts Eq.(39) of ref. [1]. Moreover, the fact that $p_{\tau}$ and $p_{1}$ are greater than one means that it is the $F^{\prime \mathrm{QFP}}$ and $F_{\tau}^{\mathrm{QFP}}$ contributions (and not the associated "1"'s!) that should be dropped out in the right-hand side of Eqs.(40)-(45) of ref. [1].

Finally, we note that comments similar to the above, hold as well in relation to the recent work [6].

\section{References}

[1] B. Ananthanarayan, P. N. Pandita, hep-ph/0011274, Phys. Rev. D63 (2001) 076008.

[2] C. T. Hill, Phys. Rev. D24 (1981) 691.

[3] G. Auberson, G. Moultaka, Eur. Phys. J. C12 (2000) 331.

[4] D.I. Kazakov, G. Moultaka, Nucl. Phys. B577 121 (2000).

[5] Y. Mambrini, G. Moultaka, M. Rausch de Traubenberg, hep-ph/0101237.

[6] P. N. Pandita, hep-ph/0103005. 


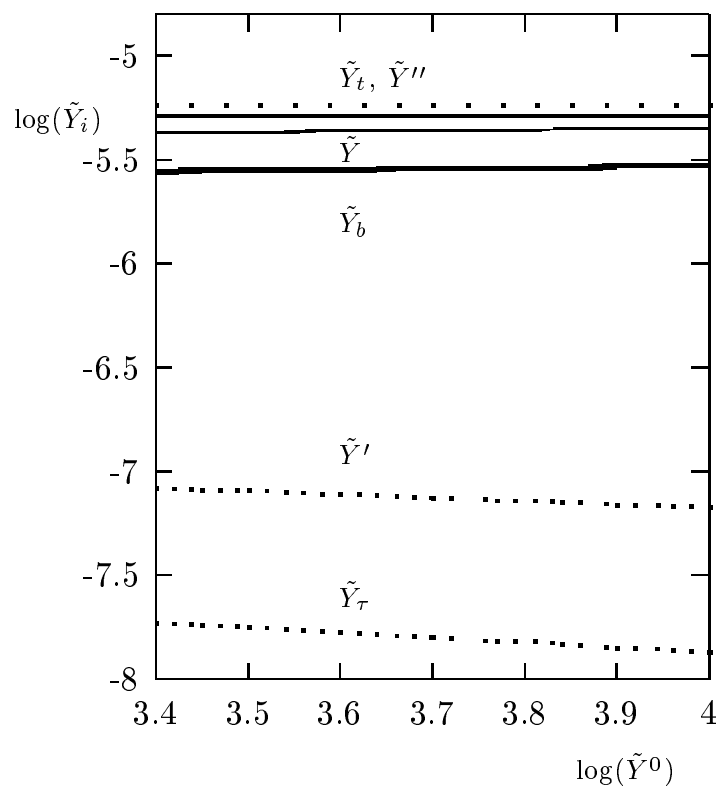

Figure 1: $\operatorname{Logs}$ of the various Yukawa couplings $\tilde{Y}_{k}(t)$ at $t=1 \mathrm{TeV}$, as a function of their common initial condition $\log \left(\tilde{Y}^{0}\right)$, where $\tilde{Y}^{0}$ takes large values in the range $30-60$. Solution (1) is clearly singled out. 